\title{
Selected Anxiety Variables of Successful and Unsuccessful Male and Female Judo Players of All India Inter-University Level
}

\author{
Sajjad Ahmad Bhat ${ }^{1 *}$, Dhananjoy Shaw ${ }^{2}$
}

\section{ABSTRACT}

The purpose of this study was to compare between male and female inter-university judo players in regard to their selected anxiety variable score at training session, during competition and at the post competition, secondly to compare between the successful and unsuccessful inter-university judo players in regard to their anxiety variable score. The study was conducted on Thirty Judo players (fifteen male and fifteen female). The subject age was ranged from 17 to 21 years. Out of thirty samples, eight male and seven female exhibited successful performances in the All India inter-university Judo competition, held at Punjabi University, Patiala 2006. Data was collected at Indira Ghandi Institute of Physical Education and Sports Sciences (IGIPESS), University of Delhi and during All India Judo championship 2006 held at Punjabi University, Patiala by administrating Illinois Competition Questionnaire (ICQ)/Competitive state Anxiety Inventory-2 (CSAI-2) following standard protocol. Descriptive statistics (Mean and standard deviation) and independent ' $t$ ' were used to analyze the data. The level of significance was set at 0.05.The finding regarding comparison of anxiety variables in CSAI-2scores of male judo players revealed that total anxiety scores were highest during post-competition stage (Mean $=67.50$ ) followed by training session $(M=59.80)$ and the total anxiety scores were lowest during competition stage (Mean =59.50). Likewise in female judo players, total anxiety were found to be highest at training session $(\mathrm{M}=70.99)$ followed by competition period $(\mathrm{M}=65.35)$ and was found lowest after the competition ( $M$ =51.32). The findings further revealed that successful judo players had higher self-confidence than unsuccessful judo players (Mean $=84.00$ ), whereas unsuccessful judo players had higher score in cognitive and somatic component of anxiety (Mean =69.33 and 59.86 respectively).

The study concluded that:-

1. During training session the male judo players scored highest in self-confidence component of anxiety followed by cognitive and somatic variable.

\footnotetext{
${ }^{1}$ Assistant Students Welfare Officer, Sher-i-Kashmir University of Agricultural Science and Technology (SKUAST-K) Srinagar, Kashmir. India

${ }^{2}$ Officiating Principal, I.G.I.P.E.S.S University of Delhi, B-Block,VikasPuri, India, New Delhi, India

*Responding Author

(C) 2016, I S Bhat, D Shaw; licensee IJIP. This is an Open Access Research distributed under the terms of the Creative Commons Attribution License (http://creativecommons.org/licenses/by/2.0), which permits unrestricted use, distribution, and reproduction in any Medium, provided the original work is properly cited.
} 


\section{Selected Anxiety Variables of Successful and Unsuccessful Male and Female Judo Players of All India Inter-University Level}

2. During training session female judo player's cognitive aspect dominated followed by selfconfidence and somatic variable.

3. During competition, cognitive variable scored highest followed by self-confidence and somatic variable in male judo players.

4. During competition female judo player's self-confidence scored highest followed by cognitive and somatic component.

5. In regard to post-competition, male judo players scored highest in self-confidence variable of anxiety followed by somatic and cognitive variable.

6. In regard to female judo players, self-confidence scored highest followed by cognitive and somatic variable of anxiety.

7. The study further revealed that successful judo players had higher self-confidence than unsuccessful judo players, whereas unsuccessful judo players had higher score in somatic and cognitive component of anxiety.

Keywords: Anxiety, Cognitive, somatic, self-confidence, Illinois Competition Questionnaire (ICQ)/Competitive state Anxiety Inventory-2 (CSAI-2)

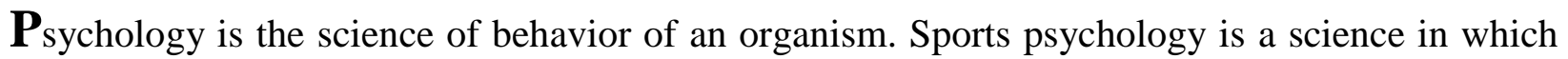
the principles of psychology are applied in a sports or exercise setting. Anxiety has been a central concept for sports psychology and has invited intensive research because of its influence on the performance. Anxiety researchers measured anxiety with general inventories such as Manifest anxiety scale (Taylor, 1953), the IPAT anxiety scale (Cattelle, 1957), Sports Competition Anxiety Test (SCAT, Martens et al.1990) and General anxiety scale (Sarason, Davidson, Lighthall, 1960). Results with these instruments led researchers to conclude that was too amorphous to be useful as predictor of behavior. Indications were that anxiety was both acute and chronic which led to the major conceptual advance best espoused by Speiberger (1966).At the same time a number of researchers were finding situation-specific anxiety scales (Watson and Friend, 1969) and within sports Martens(1977) developed the sports competition anxiety test (SCAT) which predicted anxiety state better than the other available tests, Cognitive anxiety is closely associated with worry and is defined as, "consciousness awareness of unpleasant feeling about oneself or external stimuli, worry, disturbing visual images." Somatic refers to the physiological and affective elements of the anxiety experience which develop directly from the autonomic arousal. It is reflected in response as rapid heart rate, shortness of breath, butterflies in stomach and tense muscles. (Morris et al,1981). Cognitive and somatic anxiety does influence sports performance. Somatic anxiety is likely to reach its peak at all sets of competition and dissipate once the contest begins. Thus somatic anxiety should influence performance less than the cognitive anxiety, unless somatic anxiety becomes so great that attention is diverted from task to the internal states. Cognitive anxiety is indicative of negative expectations about success 


\section{Selected Anxiety Variables of Successful and Unsuccessful Male and Female Judo Players of All India Inter-University Level}

in performing a task and these expectations are known to have powerful effects on performance. Thus Cognitive anxiety should be more strongly related to performance than the somatic anxiety. (Rosenthal, 1968, Bandura, 1977). Research revealed that total anxiety is high in combative events followed by individual events and is very low in team games. Even in the last few years study on anxiety and sports performance are constantly being conducted by the researchers (Gamit 2013, Ali Rehman 2010 and Singh 2013).

\section{Objectives}

The objective of the study was to understand the anxiety variables of All India Inter-University Male and Female Judo Players (A Comparative study) with following sub-head:

1. To compare between male and female inter-university judo players in regard to their selected anxiety variable score at training session, during competition and at the post competition.

2. To compare between the successful and unsuccessful inter-university judo players in regard to their anxiety variable score.

\section{Sample for the Study}

The study was conducted on Thirty Judo players (fifteen male and fifteen female).Ages of the sample ranged from 17 to 21 years. Out of thirty samples, eight male and seven female exhibited successful performances in the All India inter-university Judo competition, held at Punjabi University Patiala, Punjab 2006.

Table-1, Sample Distribution

\begin{tabular}{lllc}
\hline S. No & Category & Sex & Sample Size (n) \\
\hline 1 & Successful & Male & $\mathbf{8}$ \\
2 & Unsuccessful & Male & 7 \\
3 & Successful & Female & 7 \\
4 & Unsuccessful & Female & $\mathbf{8}$ \\
\hline & & & $\mathbf{N}=\mathbf{3 0}$
\end{tabular}

Data was collected at Inter-University Judo camp held at Indira Ghandi Institute of Physical Education and Sports Sciences (IGIPESS), University of Delhi and during All India Judo championship 2006 held at Punjabi University, Patiala, Punjab by administrating Illinois Competition Questionnaire (ICQ)/Competitive state Anxiety Inventory-2 (CSAI-2) followed by standard protocol. The scoring was done as per the key/guidelines recommended in the manual. Data was collected at three different stages as follows:

1. During Coaching camp/Training session(72 hours to 7 days at IGIPESS, Delhi)

2. $\quad$ During Competition (30 minutes before the bouts at Patiala, Punjab)

3. After competition (24 hours to 72 hours of competition)

(c) The International Journal of Indian Psychology, ISSN 2348-5396 (e)| ISSN: 2349-3429 (p) | 153 
Selected Anxiety Variables of Successful and Unsuccessful Male and Female Judo Players of All India Inter-University Level

\section{Statistical Analysis}

Data collected was computed using Descriptive statistics (Mean, and Standard deviation) and independent ' $t$ ' test. The level of significance was set at 0.05 .

\section{RESULT}

Table-2, Descriptive Statistics of CSAI-2 Administered on Male and Female Judo Players of All India Inter-University Judo Championship, held at Punjabi University, Patiala, Punjab

\begin{tabular}{|llllll|}
\hline Anxiety Variable & Competition Stage & Sex & N & Mean & S.D \\
& & & & & \\
Total Anxiety & Coaching Camp/ Training session & Male & 15 & 59.80 & 3.83 \\
(Cognitive/somatic/ & & Female & 15 & 70.99 & 4.38 \\
Self-confidence) & & Male & $\mathbf{8}$ & 59.50 & 1.73 \\
Competition & & Female & 7 & 65.35 & 10.37 \\
& & Male & 8 & 67.50 & 9.34 \\
Post-Competition & & Female & 7 & 51.32 & 7.48 \\
\hline
\end{tabular}

According to Table-2, it is evident that the anxiety scores of Male judo players were highest during post-competition stage (Mean $=67.50$ ) followed by training session (Mean $=59.80$ ) and was lowest during competition stage (Mean $=59.50$ ). Whereas in females, the anxiety scores were highest during Training session ( $M=70.99$ ), followed by competition ( $M=65.35$ ) and was lowest after the competition ( $\mathrm{M}=51.32$ ). The above findings have been graphically illustrated vide Figure -1 .

Figure-1

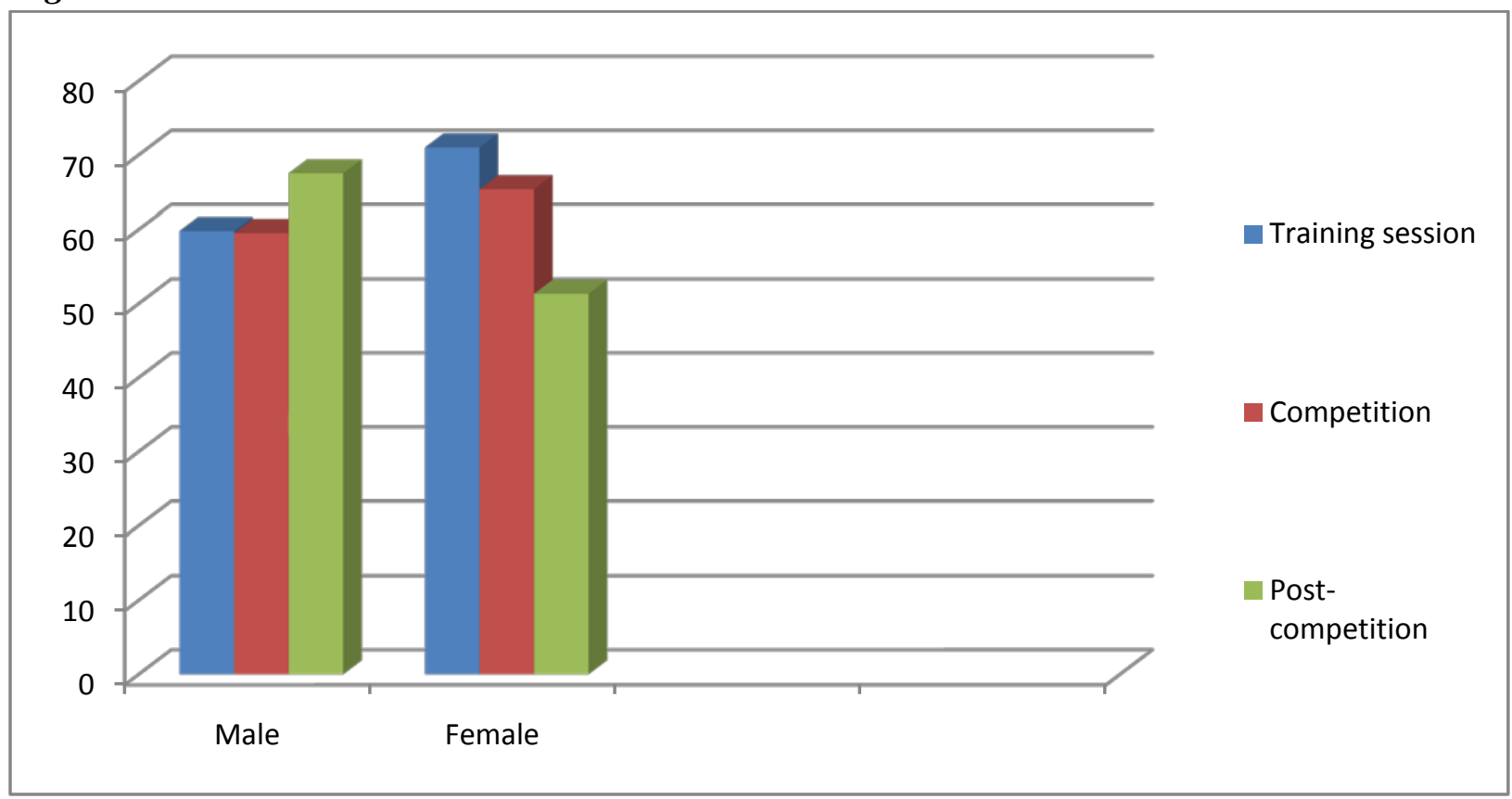

(C) The International Journal of Indian Psychology, ISSN 2348-5396 (e)| ISSN: 2349-3429 (p) | 154 
Selected Anxiety Variables of Successful and Unsuccessful Male and Female Judo

Players of All India Inter-University Level

Table-3, Comparison of CSAI-2 Components of Successful and Unsuccessful Male and Female Judo Players of All India Inter-University Judo Championship 2006 held at Punjabi University, Patiala, Punjab.

\begin{tabular}{|lclccc|}
\hline S.No & Anxiety & Category & Mean & S.D & t-value \\
\hline & Variable/Component & & & & \\
& & & & & \\
& Cognitive & Successful & 69.17 & 10.76 & 0.97 (N.S) \\
& & Unsuccessful & 69.33 & 8.43 & \\
& Somatic & Successful & 47.86 & 17.47 & 0.12 (N.S) \\
& & Unsuccessful & 59.86 & 07.70 & \\
3 & Self-Confidence & Successful & 84.00 & 13.66 & 0.17 (N.S) \\
& & Unsuccessful & 73.33 & 11.84 & \\
\hline
\end{tabular}

The analysis of the data in Table-3 pertaining to the comparison between the successful judo players and unsuccessful judo players revealed that the successful judo players having mean values for self-confidence component $(\mathrm{M}=84.00)$ than unsuccessful judo players, whereas unsuccessful judo players having higher anxiety in both somatic and cognitive component of anxiety. Further analysis revealed insignificant difference between successful and unsuccessful judo players in all the selected variables. The above findings have been graphically illustrated vide Figure -2 .

Figure-2

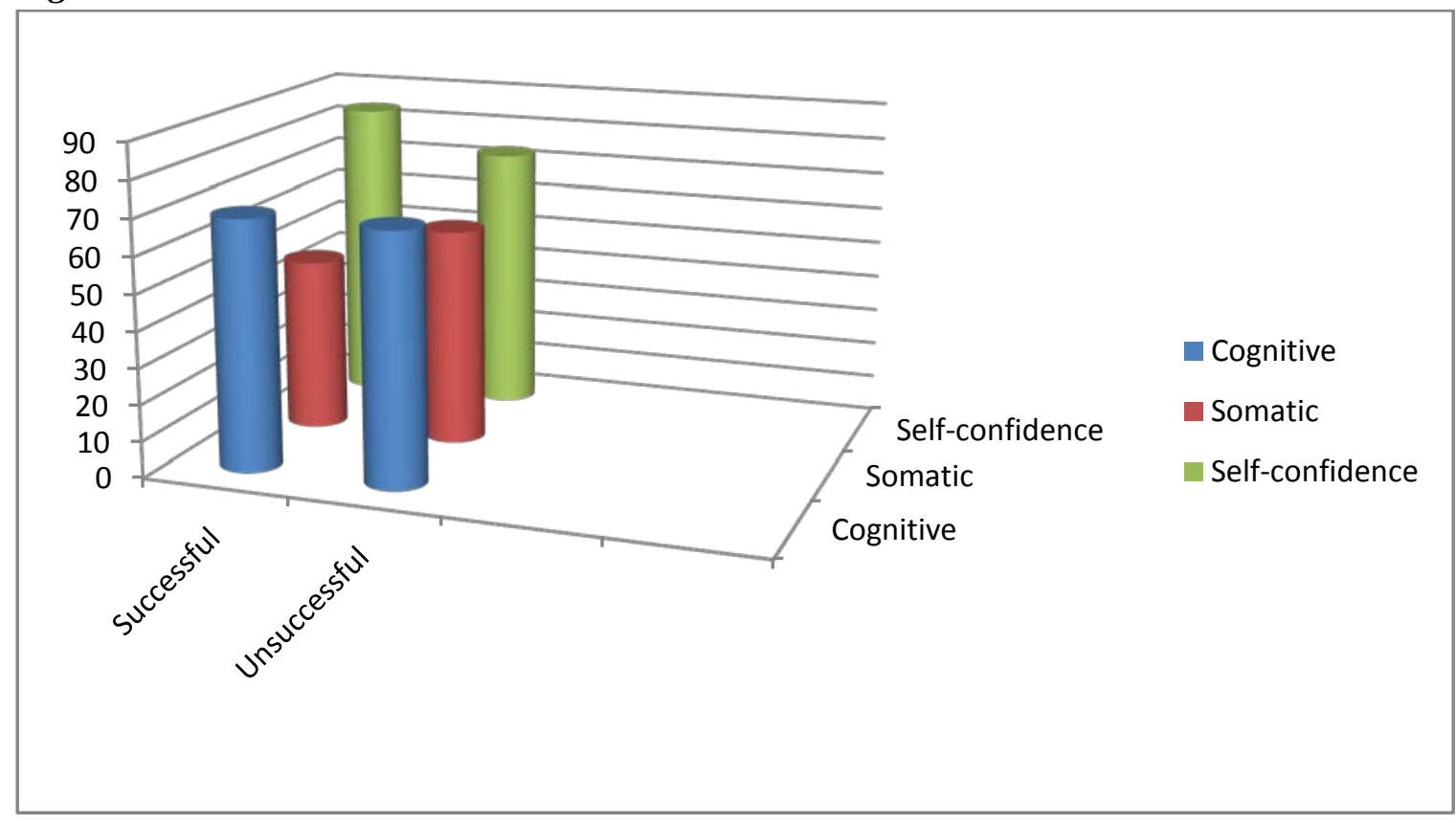

(c) The International Journal of Indian Psychology, ISSN 2348-5396 (e)| ISSN: 2349-3429 (p) | 155 


\section{Selected Anxiety Variables of Successful and Unsuccessful Male and Female Judo Players of All India Inter-University Level}

\section{DISCUSSION OF FINDINGS}

The statistical findings of the study revealed that in regard to male judo players total anxiety scores were highest during post-competition stage (Mean $=67.50$ ) followed by training session $(\mathrm{M}=59.80)$ and the total anxiety scores were lowest during competition stage (Mean =59.50). Likewise in female judo players, total anxiety were found to be highest at training session $(\mathrm{M}=$ 70.99) followed by competition period $(\mathrm{M}=65.35)$ and was found lowest after the competition ( $\mathrm{M}$ =51.32). The findings further revealed that successful judo players had higher self-confidence than unsuccessful judo players (Mean $=84.00$ ), whereas unsuccessful judo players had higher score in cognitive and somatic component of anxiety (Mean =69.33 and 59.86 respectively).

\section{CONCLUSIONS}

Within the limitations of the study following conclusions were drawn:

1. During training session the male judo players scored highest in self-confidence component of anxiety followed by cognitive and somatic variable.

2. During training session female judo player's cognitive aspect dominated followed by selfconfidence and somatic variable.

3. During competition, cognitive variable scored highest followed by self-confidence and somatic variable in male judo players.

4. During competition female judo player's self-confidence scored highest followed by cognitive and somatic component.

5. In regard to post-competition, male judo players scored highest in self-confidence variable of anxiety followed by somatic and cognitive variable.

6. In regard to female judo players, self-confidence scored highest followed by cognitive and somatic variable of anxiety.

7. The study further revealed that successful judo players had higher self-confidence than unsuccessful judo players, whereas unsuccessful judo players had higher score in somatic and cognitive component of anxiety.

\section{Acknowledgments}

The author appreciates all those who participated in the study and helped to facilitate the research process.

\section{Conflict of Interests}

The author declared no conflict of interests.

\section{REFERENCES}

Ali, J. Rehman, M. T (2010), 'A Comparative Study of Sports Competition Anxiety Between Male and Female Weight Lifters of Manipur', Human Kinetics. A Journal of Physical Education. 


\section{Selected Anxiety Variables of Successful and Unsuccessful Male and Female Judo Players of All India Inter-University Level}

Cattelle R.B, "The IPAT Anxiety Scale” Champaign II: Institute of Personality and Ability Test, 1957

Gamit A.K, "A Comparative Study of Sports Competition Anxiety Between Male and Female Cricket Players of Gujarat”. Indian Journal of Applied research,(2013),3 (1),301-302.

Martens R, “Sports Competition Anxiety Test” Campaign Il, Human Kinetics Publishers, 1977

Morris L, Davis D and Hutchings C, "Cognitive and Emotional Component of Anxiety: Literature review and revised worry-Emotional scale” journal of Educational psychology (1981)73:541-555

Rosental R, "Experimenter Expectancy and the Reassuring Nature of the Self Hypothesis Decision Procedure” Psychological Bulletin Monograph Supplement (1968):70:32-47

Sarason S. B, Davidson K.S ,Light Hall F. F, Waiter R.R and Ruebush B.K, "Anxiety in Elementary School Children”(New York: Wiley 1960)

Singh J. Jhon, S. B and Kumar A, (2013) A Comparative Study of Competitional Anxiety among Jumpers and Runners between Male and Female Athlete. International Educational eJournal,2 (5),15-17.

Spielberger C.D, Gorsuch R.L and Lushene R.E, "Manual for the state-Trait Anxiety Inventory”, (Palo Alto, CA: Consulting Psychologists Press, 1970)

Taylor J.A, "A Personality Scale of Manifest Anxiety" Journal of Abnormal and Social Psychology : (1953)285-290.

How to cite this article: S Bhat, D Shaw (2016), Selected Anxiety Variables of Successful and Unsuccessful Male and Female Judo Players of All India Inter-University Level, International Journal of Indian Psychology, Volume 3, Issue 4, No. 65, ISSN 2348-5396 (e), ISSN: 2349-3429 (p), DIP: 18.01.147/20160304, ISBN: 978-1-365-34680-4 